\title{
EDITORIAL
}

\section{Declarations of Interest}

All organizers of scientific meetings and especially International Congresses are increasingly dependent on seeking sponsors whose financial contributions are critically important for the financial security of the meeting. Sponsorship was very much in evidence at the Adelaide Congress although there was no evidence of the sponsors attempting to influence the scientific content of the programme, the sponsors being quite content for their contributions to be recognized as a public demonstration of their association with a worthy cause.

There was some criticism of the acceptance of sponsorship from some companies because of claims about their ethical behaviour. This is not the place to debate the rights and wrongs of a particular example but to focus on some points of principle in relation to publications in the scientific literature that arise from studies which have been sponsored either by a commercial organization or some other body that has an interest in the findings.

I think that it is important to consider the ethical aspects of accepting sponsorship and one wonders whether acceptance of sponsorship implies that we, as scientists, have to endorse all the activities of an industrial sponsor and, if so, which criteria should one use. For example, one could argue that selling energy-dense foods contributes to obesity, saturated fats to coronary heart disease, sugar products to dental caries, fish to the reduction of fish stocks, and dietary fibre-rich foods to the organic matter to be treated in sewage disposal. One could even argue that publication itself contributes to damage to the environment, and so on.

Furthermore, the diversification within the food industry as such would make identifying all the activities of a potential sponsor a matter for considerable research. Making objective judgements regarding the balance between activities one regards as nutritionally undesirable and those that are sound is also very difficult indeed. Especially because it is not possible to make scientific judgements about individual foods outside the context of the diet as a whole and the person eating the diet.

I think that unless the potential sponsor is making unjustifiable claims about their products or is conducting their business in an improper fashion then their contributions are acceptable, in principle, and in any case there are more competent bodies to make judgements about, and rectify, improper conduct.

Where should we draw the line when the sponsor is seeking more than publicity, on say the cover of a programme, and acknowledgement by the organizers or authors? Unacceptable demands by a sponsor would arise if there was an attempt to influence the scientific programme by, for example, insisting on the inclusion of a topic or a specific speaker. The exclusion of a particular speaker whom the organizers wished to participate on scientific grounds would be even more undesirable. Such actions would detract from the scientific integrity of the meeting.

An analogous, and possibly more serious, issue which concerns me as Editor is the submission of papers describing work which has been funded by a sponsor with a commercial or promotional interest in the findings. A situation may arise where a nutritional journal publishes a paper whose results have promotional value without proper identification of the involvement of an interested party in funding the work. The current financial situation regarding the funding of nutritional research, and indeed most scientific 
research, requires all researchers to be seeking actively for research funding, and in the nutritional field seeking collaboration with a food company is one obvious choice because such collaboration from my own experience is usually of great mutual benefit.

I believe that such collaboration is indeed essential because of our common interests in foods and their nutritional value and because dietary changes, in any direction, that the nutritional scientists may see as desirable on grounds of public health will only be achieved by a partnership between the nutritional sciences and the food producers, both primary and secondary.

Nevertheless, the scientific integrity of studies carried out with commercial or other sources of funding must be preserved at all costs and it is important to ensure that the scientific observations and the conclusions drawn from them are not influenced by the sponsors' interests. I like to believe that deliberate falsification of findings is extremely unlikely but it is possible to manipulate the conclusions that are drawn by the selective presentation of data or the omission of data that would be embarrassing or awkward. In an extreme case the submission of the paper may be delayed or never take place.

I here recognize that delay is perfectly proper where there are commercial interests of the sponsor to protect, such as seeking patents or even a production advantage from some findings which the sponsor has paid for. However, stopping publication where there are important findings of a scientific nature or with public health implications would, I believe, be unacceptable.

In my own research career I have seen examples of attempts by sponsors to veto publication because of wanting to control the release of findings which could be seen as critical, and others where work carried out without sponsorship was delayed because of concerns about upsetting the sponsor of other work. I have also experienced a commercial interest attempting to prevent work on a topic that they considered unfavourable to their interests.

It is important to remember that interests other than commercial ones can be invoked by sponsors in attempting to subvert the scientific editorial process by demanding that views that run counter to a particular line are excluded or that findings which may cause unfavourable public reaction be withheld. In my experience these types of interference are difficult to counter unless one has read the fine print of any agreement.

On the other side, I have seen sponsors acting perfectly properly when a study they had funded produced the reverse of what they had hoped and even funded a second study to confirm the findings.

I do, however, believe that, even where the sponsor of a particular study has made no attempts to interfere with the scientific integrity of the study and the publication of the paper describing the work, it is important for such sponsorship to be noted in the paper, as indeed it usually is. Where a sponsor has exerted control over the textual content of a paper one could argue that a representative of the sponsor should be listed as an author.

We, as researchers, can only benefit from collaboration with the food industry but I believe that all sponsors must respect the scientific integrity and independence of the nutritional scientists they choose to work with, because without these the value of the collaboration would be compromised. Scientific sycophancy is, I believe, as worthless for the sponsors as it is for the scientists.

D. A. T. Southigate 